\title{
BMJ Global Health Interventions to support family caregivers of people living with dementia in high, middle and low- income countries in Asia: a scoping review
}

\author{
Ladson Hinton (D) , ${ }^{1}$ Duyen Tran, ${ }^{2}$ Thuc-Nhi Nguyen, ${ }^{3}$ Janis Ho, ${ }^{4}$ Laura Gitlin ${ }^{5}$
}

To cite: Hinton L, Tran D, Nguyen T-N, et al. Interventions to support family caregivers of people living with dementia in high, middle and low-income countries in Asia: a scoping review. BMJ Global Health 2019;4:e001830. doi:10.1136/ bmjgh-2019-001830

Handling editor Seye Abimbola

Received 10 July 2019

Revised 25 September 2019

Accepted 28 September 2019

Check for updates

(C) Author(s) (or their employer(s)) 2019. Re-use permitted under CC BY-NC. No commercial re-use. See rights and permissions. Published by BMJ.

${ }^{1}$ Department of Psychiatry and Behavioral Sciences, University of California Davis School of Medicine, Sacramento, California, USA

${ }^{2}$ University of California Davis, Davis, California, USA

${ }^{3}$ Columbia University, New York City, New York, USA

${ }^{4}$ Touro University California, Vallejo, California, USA

${ }^{5}$ College of Nursing and Health Professions, Drexel University, Philadelphia, Pennsylvania, USA

Correspondence to

Dr Ladson Hinton;

Iwhinton@ucdavis.edu

\section{ABSTRACT}

Introduction Despite increasing numbers of persons living with Alzheimer's disease and Alzheimer'srelated dementias (AD/ADRD) in Asia, particularly in low-income countries (LIC) and middle-income countries (MIC), surprisingly little is known about the current state of the evidence for family caregiver interventions. The objectives of this scoping review were to: (1) describe the evidence for efficacy of family dementia-caregiver psychosocial interventions in Asian countries, (2) compare evidence across LIC, MIC, and high-income countries (HIC), and (3) characterise cultural adaptions to interventions developed outside Asia.

Methods The inclusion criteria included: (1) conducted in Asia (2) included an intervention delivered to a family caregiver of a person living with AD/ADRD, (3) reported quantitative outcomes for the family caregiver and (4) published in a peer-reviewed journal with full text available in English.

Results Thirty intervention trials were identified meeting inclusion criteria and all reported statistically significant $(p<0.05)$ improvement in one or more caregiver outcomes. Interventions usually included multiple components. The most frequently reported outcomes (ie, by $\geq 20 \%$ of studies) were caregiver depression, burden, quality of life and self-efficacy. Overall, $26(87 \%)$ of the studies were conducted in HIC in Asia, primarily in Hong Kong SAR-China and Taiwan, and only $4(13 \%)$ in LIC and MIC in Asia. Seven studies (23\%) used interventions developed in USA and several described cultural adaptations.

Conclusion This scoping review found substantial evidence, particularly from high-income Asian countries, that a wide range of interventions improve AD/ADRD family caregiver outcomes. However, critical knowledge gaps exist, particularly for LIC and MIC in Asia, where the number of persons with dementia is numerically largest and projected to increase dramatically in coming decades. The field could also benefit from more detailed descriptions of the process and types of cultural adaptations to interventions.

\section{Key questions}

What is already known?

- The number of older persons in Asia with Alzheimer's disease and Alzheimer's-related dementias (AD/ ADRD) will increase substantially in the coming decades, particularly in low-income countries (LIC) and middle-income countries (MIC).

- Family caregivers of persons with dementia may be at increased risk for adverse mental health and health outcomes.

- Multicomponent interventions have been shown to improve caregiver outcomes in USA and Europe but the state of the evidence in Asia is not known.

What are the new findings?

- Thirty intervention studies were identified and all reported significant improvement in one or more caregiver outcomes, such as depression, burden, quality of life, self-efficacy.

$70 \%$ of the studies were conducted in 2 high-income countries (HIC) (Hong Kong SAR-China and Taiwan) and only $13 \%$ (4) in LIC and MIC.

What do the new findings imply?

- Multicomponent interventions are a promising approach to improve outcomes for AD/ADRD family caregivers in Asia.

- There is an urgent to close the striking gap in evidence, particularly for low and middle-income countries

\section{INTRODUCTION}

Asia includes some of the most populous (eg, China and India) and rapidly ageing societies in the world. ${ }^{12}$ Together with the growth in their older adult populations, countries in Asia will experience a dramatic increase in the number of people living with Alzheimer's disease and Alzheimer's-related dementias (AD/ADRD), particularly in lowerincome countries (LIC) and middle-income 
countries (MIC). It is projected that from 2015 to 2050 LIC and MIC worldwide will see the number of people living with $\mathrm{AD} / \mathrm{ADRD}$ triple versus a doubling in highincome countries (HIC). ${ }^{3} \mathrm{AD} / \mathrm{ADRD}$ are among the most disabling diseases to affect older adults and have negative health and economic consequences for family members who often are relied on to provide care and support. To prepare for the growing number of older adults with $\mathrm{AD} / \mathrm{ADRD}$ and caregivers, it is important for countries in Asia to develop and strengthen communitybased programmes and supports for family caregivers.

Although dementia is not curable, a large and growing evidence-base exists in western HIC for nonpharmacological interventions to improve caregiver outcomes. ${ }^{45}$ Common elements of effective interventions include caregiver education, skill-building to manage difficult behavioural problems and referral to community resources. Despite the growing number of persons living with $\mathrm{AD} / \mathrm{ADRD}$ in Asia, little is known about the current state of the evidence for family caregiver interventions in these countries. This knowledge is important for both research and policy makers as they plan for ageing populations and the increasing need to support family caregivers using the best practices in the most effective and cost-efficient manner.

We conducted a scoping review to address this gap in our knowledge about the evidence base for $\mathrm{AD} / \mathrm{ADRD}$ family caregiver interventions in Asia. The specific objectives of this scoping review of the literature were to: (1) describe and map the existing evidence for efficacy or effectiveness of family dementia-caregiver psychosocial interventions in countries in Asia (broadly defined to include 20 countries in East Asia, South Asia and Southeast Asia), (2) compare the availability of evidence across LIC, MIC, and HIC Asian countries, and (3) for those interventions that were developed outside Asia, to characterise the types of adaptations, cultural or otherwise. A scoping review is designed to 'scope' a broad body of literature to identify knowledge gaps and summarise the available evidence. To conduct the review, we followed accepted guidelines for the scoping review process. ${ }^{6}$ For this review, we focused on 20 countries in Asia (see under methods below for full list) that included the two most populous countries in the world (China and India) and three additional countries that are among the top ten in terms of population size (Pakistan, Indonesia, Bangladesh). These countries are also socioeconomically diverse and include HIC, MIC and LIC, thus affording country comparisons by income level.

\section{METHODS}

\section{Study inclusion and exclusion criteria}

Studies were included that met the following criteria: (1) conducted in one or more of the 20 countries in East Asia, South Asia or Southeast Asia (multi-site studies that included and reported outcomes for at least one site located in one of the 20 countries were also included),
(2) intervention with one or more components delivered to a family caregiver (defined broadly to include family, friends, neighbours) of a person living with $\mathrm{AD} / \mathrm{ADRD}$, (3) reported quantitative outcomes for family caregivers and (4) published in a peer-reviewed journal with full text available in English. When multiple articles were published from a single intervention trial, we grouped them together. Based upon the 2018 World Bank classification, the 20 selected countries include $6(30 \%)$ from HIC (Hong Kong SAR—China, Taiwan, Singapore, Korea, Japan), 3 (15\%) from upper MIC (Thailand, Mainland China, Malaysia), $10(50 \%)$ from lower MIC (Vietnam, Bhutan, Mongolia, Bangladesh, Sri Lanka, Myanmar, Indonesia, India, Cambodia, Philippines) and 1 from LIC (Pakistan).

\section{Search methods}

A review was conducted of literature published from inception through June of 2018 in Embase, PsychInfo, PubMedand Scopus. Search terms included ('alzheimer disease' OR alzheimer's OR 'dementia, vascular' OR dementia OR FTD OR lewy body OR 'lewy bodies') AND ('caregivers/psychology' OR caregiver) AND (mental health OR support OR social OR environmental OR education OR programs OR strategies OR services OR care services OR supportive services OR intervention OR process assessment OR outcomes assessment)) AND ('asian continental ancestry group' OR asian OR indian OR india OR pakistani OR pakistan OR bangladeshi OR bangladesh OR sri lankan OR sri lanka OR bhutanese OR bhutan OR nepalese OR nepal OR japanese OR japan OR vietnamese $O R$ viet nam OR vietnam OR filipino OR philippines OR burmese OR burma OR cambodian OR cambodia OR thai OR thailand OR korean OR korea OR mongolian OR mongolia OR taiwanese OR taiwan OR hong kong OR chinese OR china OR malaysian OR malaysia OR indonesian OR indonesia OR singapore). Also included were articles that had been identified by members of the study team.

We used several steps to identify the studies included in this scoping review, using EndNote to manage the citations. Once all articles were transferred to EndNote, we removed duplications, books, book chapters, theses and articles with no titles. Following this, we also examined titles and eliminated irrelevant articles. Once irrelevant articles were removed, abstracts of the remaining records were downloaded and two research assistants independently reviewed the abstracts to determine their fit with inclusion criteria, and then met to compare results. A third reviewer provided feedback when the two research assistants could not reach an agreement to resolve discrepancies. For the remaining abstracts that were determined to have met study criteria, full-length articles were pulled and reviewed independently for their relevance to the scoping review by two research assistants with differences resolved by a third-party review. 


\section{Abstracting and charting}

For articles meeting inclusion criteria, we abstracted information and charted characteristics from the fulllength articles. Abstracting was done independently by two research staff who then compared their results and resolved any discrepancies in consultation with the first author. The information that was abstracted included article title and authors, country with income level, ${ }^{7}$ as well as intervention total sample size, name and components, duration, design, comparison group (if applicable) and outcomes reported with results. Studies emanating from the same intervention trial were grouped together to generate a list of unique intervention trials. For the summary of evidence by country income level, we used the 2018 World Bank classification of country income. ${ }^{7}$ We abstracted information on sociocultural adaptations for those studies that referenced use of a specific intervention model developed in the USA or in another country.

\section{Patient and public involvement}

There was no involvement of patients or the public in this scoping review.

\section{RESULTS}

\section{Search results}

As shown in figure 1, 13572 articles were identified across the four databases (4099 in Pubmed, 115 in Scopus, 7953 in PsycInfo and 1405 in Embase). An additional 34 records were identified through members of the research team. After removal of duplicate articles and screening of titles, the abstracts of the remaining 406 articles were further assessed for eligibility. A total of 41 abstracts meeting inclusion and exclusion criteria were identified and full-length articles were then obtained. Based on review of the full-length articles, six articles were found not to meet study criteria, leaving a total of 35 articles. The 35 publications were based on 30 unique clinical intervention trials, with two of the trials generating multiple publications. ${ }^{8-42}$

\section{Intervention characteristics}

The 30 unique intervention trials meeting full inclusion criteria and their characteristics are summarised in table 1 . More than half of the trials were conducted in Hong Kong SAR—China (17), followed by Taiwan (4), Japan (2), South Korea (2), and one each from mainland China, India, Pakistan, Singapore (multi-site study), and Thailand. Study sample sizes (based on sample size analysed) ranged from 8 to 201 family caregivers. Across all intervention studies a total of 1811 family caregivers participated. Study duration ranged from 4 weeks to 12 months with nearly all studies (29/30) being 6 months or less. In terms of design, 22 were randomised controlled trials and 8 were clinical trials that did not include random allocation. Almost all studies were multicomponent interventions (see table 1 for a list of the primary components in each of the 30 intervention studies) that often included several of the following in combination: psychoeducation, caregiver stress reduction and self-care, caregiver skill-building and coping, pleasant event scheduling, support groups, home/environmental modifications and information about community services. Of the 30 studies, 7 referenced use of specific multicomponent interventions developed in another country (in all cases in the USA) such as Resources for Enhancing Caregiver Health (REACH II) ${ }^{43}$ and Coping with Caregiving. ${ }^{4}$

\section{Efficacy of interventions}

A wide range of caregiver outcome measures were used in the intervention trials and most reported multiple caregiver outcomes (intervention trials whose results are reported in multiple publications are presented in aggregate). Table 2 summarises the results for most commonly reported caregiver outcomes (eg, for $20 \%$ or more of the intervention trials), which included burden, depression, caregiver quality of life and selfefficacy (outcomes for persons living with dementia were not included). All 30 intervention trials reported statistically significant $(\mathrm{p}<0.05)$ improvement in one or more of these most commonly reported caregiver outcomes. For each of these outcomes, between $57 \%$ (self-efficacy) and $100 \%$ (caregiver quality of life) of the studies in which they were reported found statistically significant improvement $(\mathrm{p}<0.05)$. Depression was the

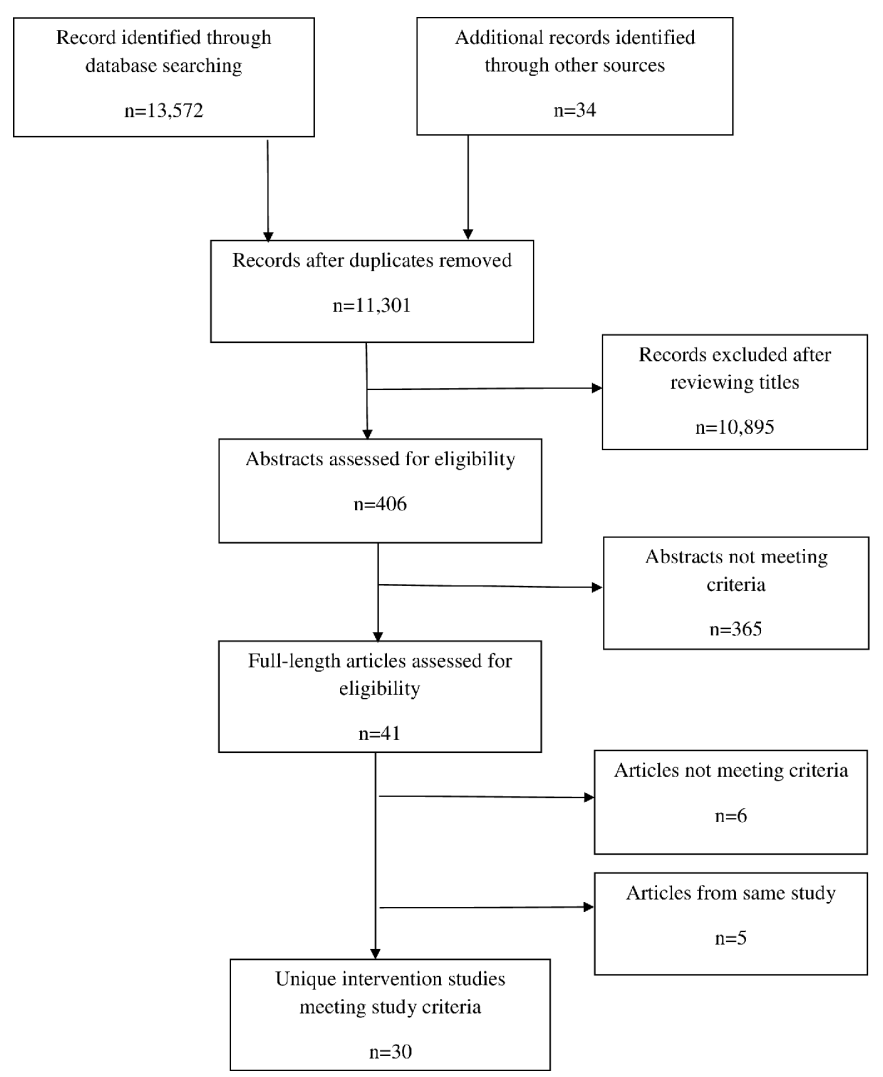

Figure 1 Selection process for scoping review. 


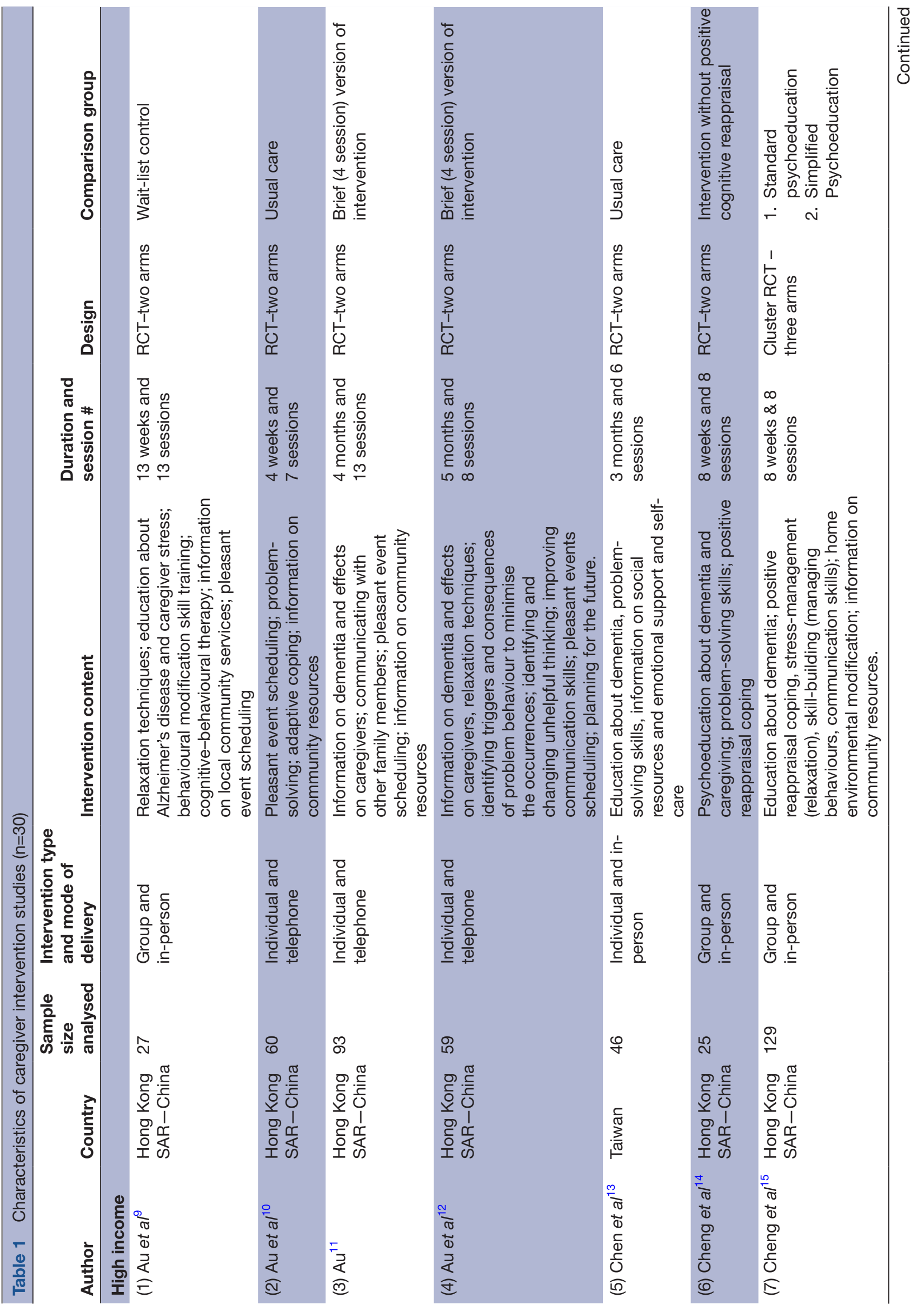




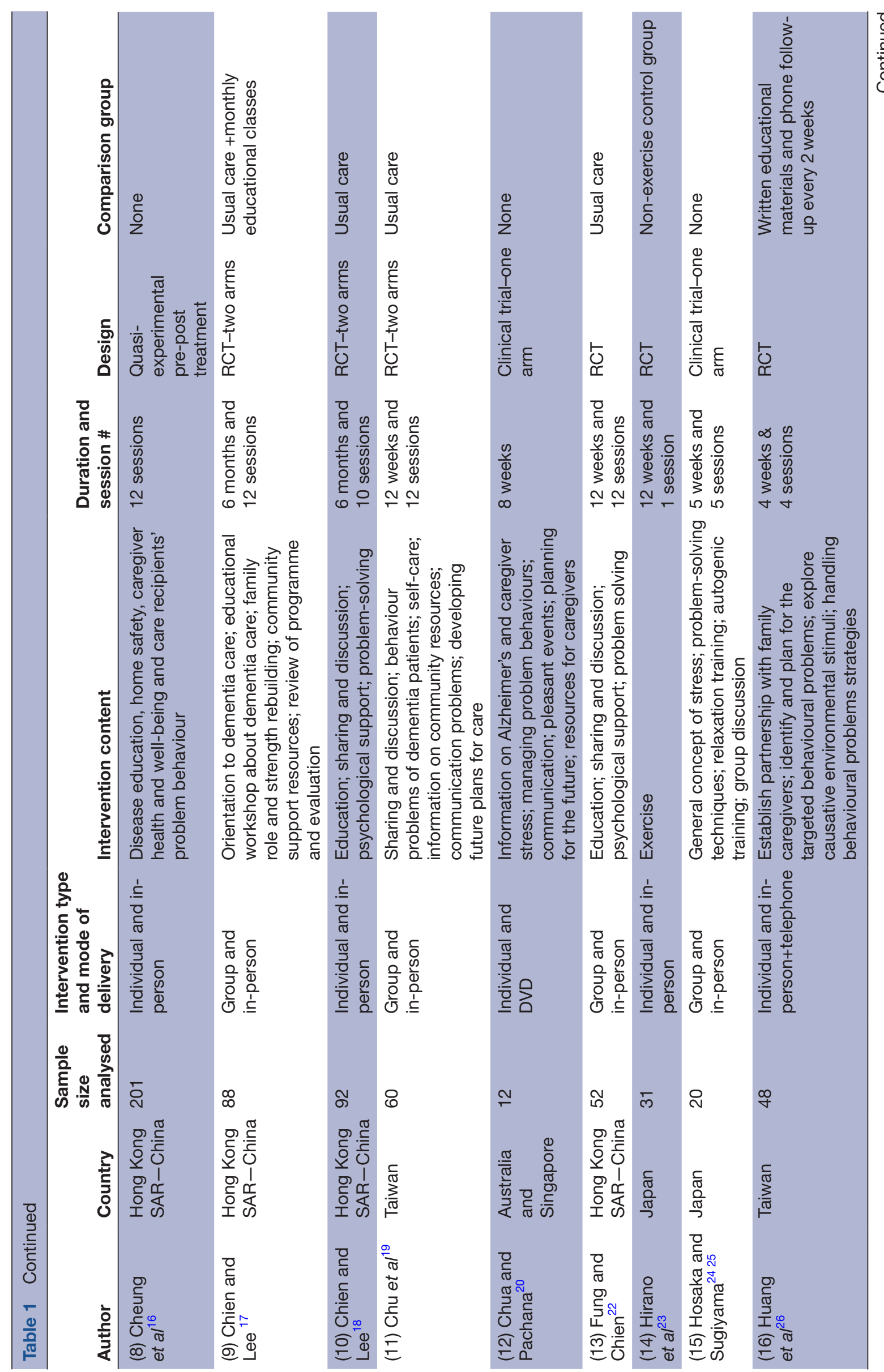




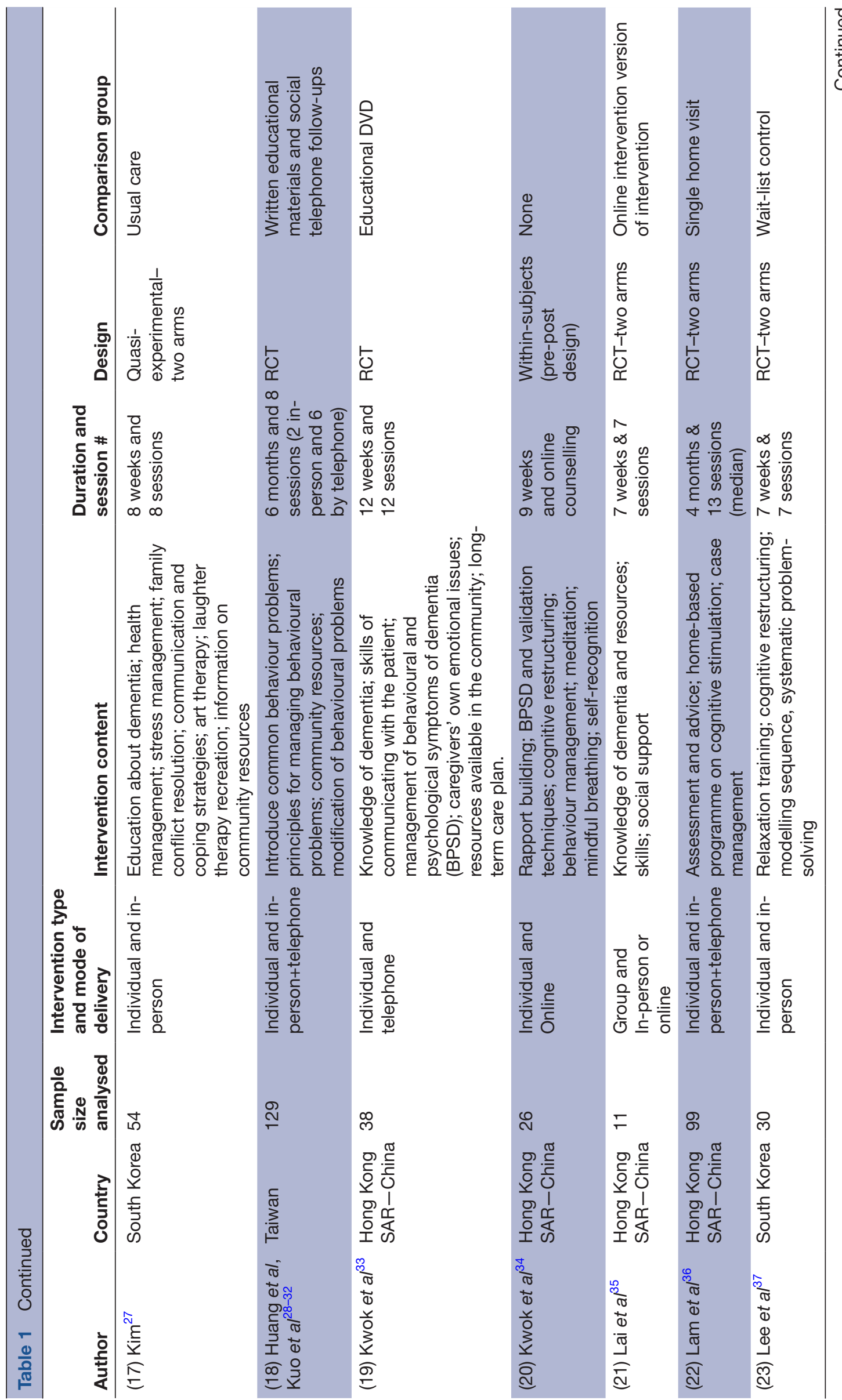




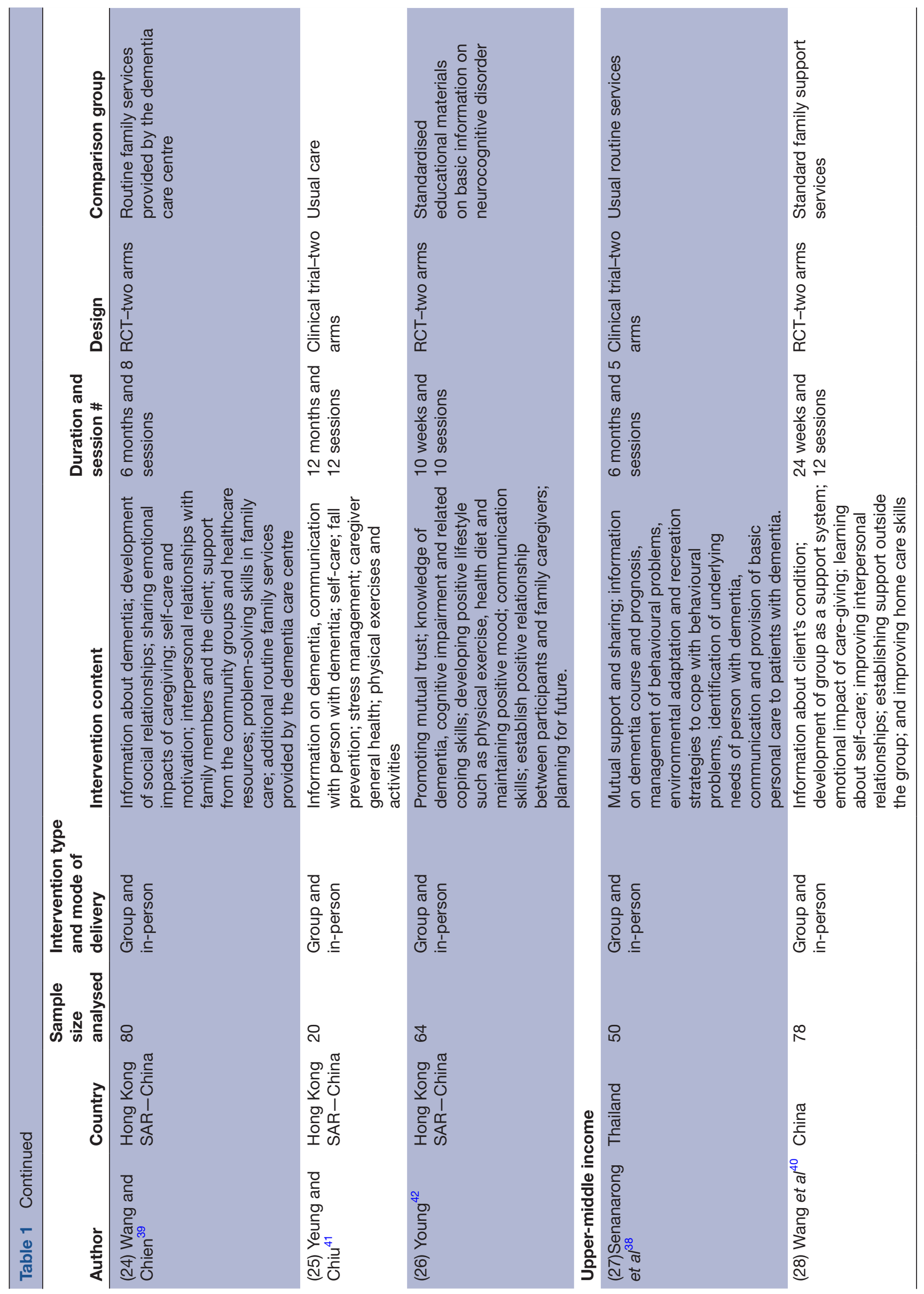




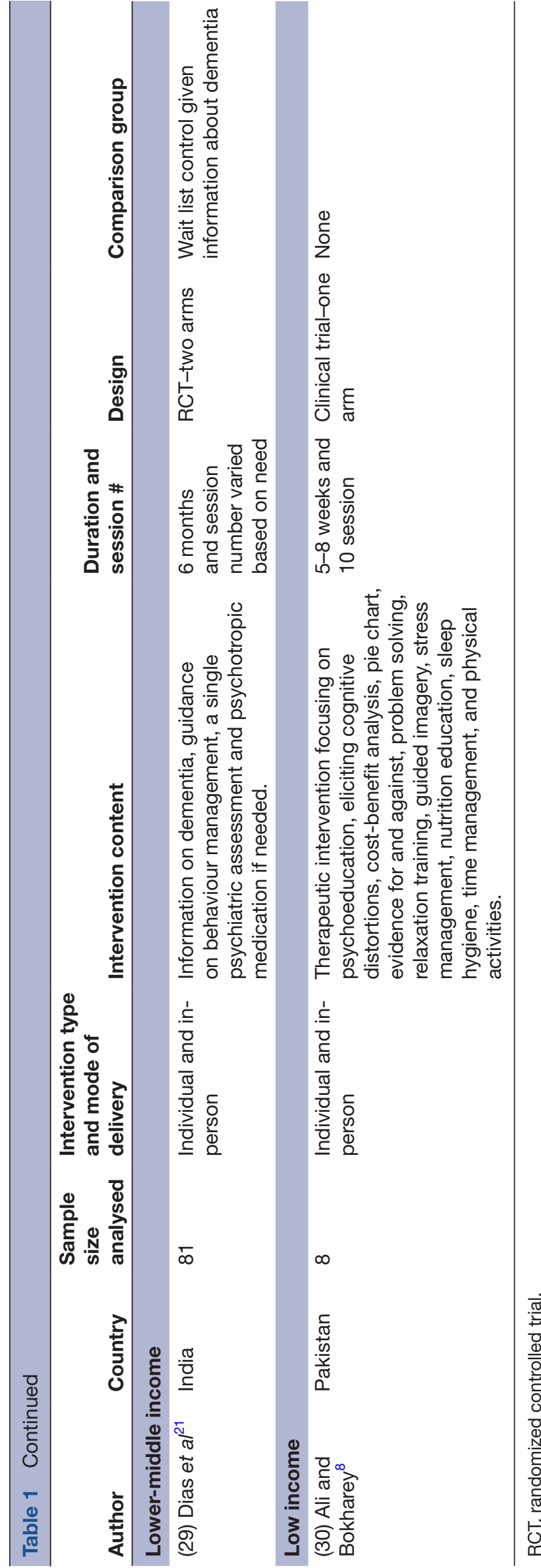

most commonly reported outcome (ie, $63 \%$ or $19 / 30$ studies).

\section{Evidence by country income level}

In table 3 we summarise the availability of evidence by country income level using the World Bank Classification (2018) using both number of individual studies and the total number of participants across all individual studies. Overall $87 \%$ of the intervention trials $(n=26)$ were conducted in five HIC (Hong Kong SAR-China, Taiwan, Japan, Singapore, South Korea), $7 \%(\mathrm{n}=2)$ in upper MIC (mainland China, Thailand), 3\% $(\mathrm{n}=1)$ in lower MIC (India) and 3\% ( $\mathrm{n}=1)$ in a LIC (Pakistan). We also tabulated the total number of participants in the studies conducted in each of the different country income levels (see table 3). The number of participants ranged from a total of 1594 in HIC to 8 in LIC. Overall, $88 \%(n=1594)$ of the total trial participants $(n=1811)$ were from five HIC.

\section{Cultural adaptations to interventions}

While many studies based their interventions on theoretical models developed outside Asia, only seven studies reported using specific intervention models developed outside Asia. A study by Cheung et $a l^{16}$ described several adaptations to the REACH II model, including translating all materials (manuals, protocol and caregiver notebook) into Chinese using bilingual assistants, conducting most sessions at the local service agency because of limited space in caregiver's homes, omitting group sessions due to resource constraints and logistic reasons and not adopting a specialised computer-integrated telephone system. In addition, changes were made to the inclusion criteria and in the battery of instruments administered compared with the original REACH II study. Four studies $^{9-12}$ were based on the Coping with Caregiving Intervention. ${ }^{44}$ Of those, one noted adding an additional outcome measure to assess coping flexibility ${ }^{9}$ while another ${ }^{12}$ reported making several adaptations, including emphasising behavioural activation rather than changing dysfunctional thoughts to reflect cultural values, overcoming barriers to participation by delivering the intervention by phone, and training and using paraprofessionals to deliver the intervention to enhance sustainability. A programme for using a cognitive-behavioural therapy DVD intervention to treat caregivers adapted by Chua and Pachana ${ }^{20}$ reported several minor modifications, including replacing content related to American statistics, slight language editsand omitting a chapter on planning for the future. A final study by Chu et $a l^{19}$ was based on a caregiver support programme and manual developed by Zarit and colleagues but did not provide any information about adaptations.

\section{DISCUSSION}

As the population of persons living with dementia increases throughout Asia, understanding the ways to best support family caregivers is critical. This scoping 
Table 2 List of commonly reported caregiver outcomes and results

\section{Burden Depression Quality of life Self-efficacy Other*}

High income

\begin{tabular}{|c|c|c|c|c|c|}
\hline (1) Au et $a l^{9}$ & & - & & + & + \\
\hline (2) Au et $a l^{10}$ & & + & & - & \\
\hline (3) $A u^{11}$ & & + & & & + \\
\hline (4) Au et $a l^{12}$ & & + & & & + \\
\hline (5) Chen et al ${ }^{13}$ & + & & & & + \\
\hline (6) Cheng et $a^{14}$ & - & + & & & - \\
\hline (7) Cheng et $a l^{15}$ & + & + & & + & + \\
\hline (8) Cheung et $a l^{16}$ & + & + & & & + \\
\hline (9) Chien and Lee ${ }^{17}$ & + & & + & & \\
\hline (10) Chien and Lee ${ }^{18}$ & + & 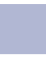 & + & & \\
\hline (11) Chu et $a l^{19}$ & - & + & & & \\
\hline (12) Chua and Pachana ${ }^{20}$ & & - & & & + \\
\hline (13) Fung and Chien ${ }^{22}$ & & - & + & & - \\
\hline (14) Hirano et $a l^{23}$ & + & - & & & + \\
\hline (15) Hosaka and Sugiyama 2425 & & + & & & + \\
\hline (16) Huang et $a^{26}$ & & & & + & \\
\hline (17) $\mathrm{Kim}^{27}$ & & + & & & \\
\hline (18) Huang et al, Kuo et $a l^{28-32}$ & & + & + & + & + \\
\hline (19) Kwok et al ${ }^{33}$ & + & & & - & \\
\hline (20) Kwok et al ${ }^{34}$ & + & & & - & \\
\hline (21) Lai et $a l^{35}$ & & + & + & & + \\
\hline (22) Lam et a/ $/^{36}$ & - & + & & & + \\
\hline (23) Lee et $a /^{37}$ & + & + & & & + \\
\hline (24) Wang and Chien ${ }^{39}$ & + & & + & & \\
\hline (25) Yeung and Chiu ${ }^{41}$ & + & & & & \\
\hline
\end{tabular}

\section{Upper-middle income}

(27) Senanarong et $a l^{38}$

(28) Wang et al ${ }^{40}$

\section{Lower-middle income}

(29) Dias et $a l^{21}$

\section{Low income}

(30) Ali and Bokharey ${ }^{8}$

Notes: + indicates a significant improvement $(p<0.05)$ in outcome, - is a lack of significant improvement $(p>0.05)$, blank indicates outcome not reported.

${ }^{*}$ Caregiver outcomes present in $<20 \%$ of studies.

review, the first of its kind, maps the available evidence for interventions to support family caregivers of persons living with dementia in Asia and the distribution of evidence by country income level using the World Bank's classification. Several important findings emerge from this scoping review and suggest ways to advance the field.

Despite heterogeneity in the characteristics of the interventions (eg, specific intervention components, type, duration), nearly all studies included in this review reported statistically significant improvement in one or more outcomes of importance to the well-being of family caregivers. Our results are consistent with a recent systematic review of dementia caregiving interventions in Chinese people, which also found generally positive results and which included a subset of the studies that were included in our review. ${ }^{45}$ Also of importance is that positive outcomes were found for HIC, MIC and LIC suggesting that families regardless of country income level and resources derive important benefits from psychosocial supportive interventions. There is 
Table 3 Number of intervention studies and participants (analysed) by country income level

\begin{tabular}{lcc}
\hline & Studies $\mathbf{( n )}$ & $\begin{array}{l}\text { Total } \\
\text { number of } \\
\text { caregivers }\end{array}$ \\
\hline High income & 26 & 1594 \\
Upper-middle income & 2 & 128 \\
Low-middle income & 1 & 81 \\
Low income & 1 & 8 \\
\hline
\end{tabular}

considerable overlap in the components included in interventions, which typically included caregiver education (Alzheimer's disease, caregiving issues, community resources), stress-reduction and skill-building. Overall, our findings are encouraging and suggest that multicomponent interventions and a variety of psychosocial intervention approaches may be effective in Asia even though most were based on theoretical models or specific intervention approaches developed outside of Asia.

There may be several reasons that the seven studies using intervention models initially designed in USA were also effective for caregivers in Asia. First, interventions tested in the reviewed studies were delivered by local professionals in the language of the targeted population and modified in a variety of ways to meet the needs of the local population, which may have enhanced their cultural acceptability. Second, interventions developed with US samples tend to involve tailoring to meet different needs of caregivers. This flexibility and personalisation in content and delivery may optimise transferability of interventions from one country and culture to another. Finally, the efficacy of intervention models developed outside Asia suggests substantial cross-cultural similarities in the nature and sources of caregiver stress and the kinds of approaches that are successful in alleviating caregiver suffering and improving knowledge, skills and coping abilities.

A second major finding from this review is that the evidence is very unevenly distributed in terms of country income levels, with more than two-thirds of the evidence (ie, 21/30 studies) coming from just two HIC (Hong Kong SAR-China and Taiwan) and very little data coming from MIC and LIC. Of the 30 intervention trials, only four were conducted in MIC or LIC. Equally striking was the disparity in the number of participants in studies in HIC compared with MIC and LIC-of the more than 1800 participants across all trials included in this review, only $13 \%$ were from MIC and LIC countries. This gap in our knowledge about the efficacy and effectiveness of interventions in lower MIC and LIC is particularly striking and disconcerting considering that MIC and LIC countries are together more populous and projected to experience the greatest increase in persons living with dementia in coming decades. By 2030, over $70 \%$ of the people with $\mathrm{AD} / \mathrm{ADRD}$ will live in MIC and LIC. ${ }^{46}$ Even among the HIC, there were surprisingly few studies outside of Hong Kong SAR — China and Taiwan. While the lack of studies in LIC and MIC might plausibly reflect lack of sufficient resources and infrastructure to conduct intervention trials, the reasons for the dearth of studies in HIC such as Japan or Korea as well as mainland China are less clear, although it is possible that additional intervention studies have been published in languages other than English but were excluded because of our study inclusion criteria.

While many of the studies included in this review were based on theoretical models and intervention approaches originating outside Asia, only a handful reported adapting specific intervention models. The adaptations reported were relatively few and fell primarily into the categories of language (ie, translation of manuals, protocols, measures and caregiver materials into Chinese), content (eg, including tailoring materials to reflect local resources, omitting aspects of the interventions that were not feasible or culturally well-accepted, and introducing culturally-salient topics), structure (ie, delivery of the intervention in community agencies rather than the home due to space concerns, shortening the number of sessions) and interventionist training (eg, training paraprofessionals to expand reach). As more intervention studies are conducted in lower MIC and LIC, describing in more detail the types of adaptations and related costs in relationship to local socioeconomic circumstances, which may vary within country, will be particularly important for sustainability and scalability. Many older adults in LIC and MIC, for example, reside in rural areas where the population is poorer and there is less access to supports and services. In addition, the use of adaptation frameworks, such as Bernal's ${ }^{48}$ to plan and structure the adaptation process may be useful to guide cultural adaption of evidence based interventions.

Some limitations of this scoping review should be noted. Perhaps the most important is that this review was limited to articles that were published and available in English. This may have excluded studies that were published in other languages thus underestimating the amount of evidence. In addition, consistent with the goals of a scoping review, we did not attempt to assess the quality of the evidence which is likely to be highly variable across studies. Finally, we limited our review to studies of $\mathrm{AD} / \mathrm{ADRD}$ caregiving which may have excluded interventions to support family caregivers of older adults with a broad range of chronic health conditions not limited to dementia.

Nevertheless, this scoping review has identified important directions for future work. Studies could be strengthened by more attention to the validity of the outcome measures for the target population as well as to mechanisms of action, or why an intervention has a benefit. One urgent need is for the field to develop evidence from a wider range of countries, particularly from MIC and LIC which will experience a disproportionate increase in the number of persons living with dementia in the coming decades. Studies are underway 
to address this gap in India ${ }^{49}$ and Vietnam ${ }^{50}$ but much more evidence is needed from these and other Asian MIC and LIC countries. From an intervention standpoint, the evidence base in Asia is limited to efficacy studies, with little data available on other types of intervention studies, such as pragmatic trials. Because pragmatic trials test the effectiveness of interventions under conditions that more closely approximate usual care, they can be particularly useful in generating evidence necessary for broader dissemination, implementation and policy change. ${ }^{51} 52$ The field might benefit from the use of existing theoretical frameworks and implementation science to guide the adaption process along with more detailed description of modifications to measures, manuals and other related materials to reflect local cultural concepts, content and metaphors. Additional details about the adaptation process, to both local socioeconomic and care delivery contexts, is critical to inform efforts to develop, implement and scale-up family dementia caregiving interventions in Asia. Future work may also want to consider whether findings and lessons learnt from intervention studies conducted with Asian caregivers residing in countries outside of Asia (eg, the USA) can help to strengthen caregiver interventions in Asia (and vice versa).

\section{CONCLUSION}

This scoping review found substantial evidence, particularly from HIC in Asia, that a wide range of psychosocial caregiver interventions improve caregiver outcomes. However, there are critical gaps in our knowledge, particularly for MIC and LIC in Asia, where the number of persons with dementia is numerically largest and which are anticipated to experience the most dramatic future growth in the number of persons living with dementia. From a public health perspective, there is an urgent need to develop and test caregiver interventions in MIC and LIC that have the potential to be scaled-up.

Acknowledgements The authors thank Mai Nguyen and Eduardo Delgadillo Alfaro for assistance in screening and reviewing abstracts.

Contributors LH had primary responsibility for study design, overseeing data acquisition and interpretation, and drafting the manuscript. DT, JH, and T-NN made substantial contributions to data acquisition, interpretation and drafting key parts of the manuscript. LG made substantial contributions to conceptualising the study, interpreting the data and making critical revisions to drafts of the manuscript. All authors approved the final version of the manuscript and agree to be responsible for all aspects of the work.

Funding Research reported in this publication was supported by National Institute on Aging of the National Institutes of Health under award numbers R21 AG054262 (Dr. Hinton PI). LG was supported by National Institute on Aging grants R01 AG049692 and R01 AG041781.

Disclaimer The content is solely the responsibility of the authors and does not necessarily represent the official views of the National Institutes of Health.

Competing interests None declared.

Patient consent for publication Not required.

Ethics approval Because this was a review of the published literature (ie, a scoping review) and did not involve human subjects, IRB approval was not necessary.

Provenance and peer review Not commissioned; externally peer reviewed. Data availability statement Data are available upon reasonable request.
Open access This is an open access article distributed in accordance with the Creative Commons Attribution Non Commercial (CC BY-NC 4.0) license, which permits others to distribute, remix, adapt, build upon this work non-commercially, and license their derivative works on different terms, provided the original work is properly cited, appropriate credit is given, any changes made indicated, and the use is non-commercial. See: http://creativecommons.org/licenses/by-nc/4.0/.

\section{ORCID iD}

Ladson Hinton http://orcid.org/0000-0001-6198-8540

\section{REFERENCES}

1 Work Bank Group. Live long and prosper: aging in East Asia and the Pacific, 2016. Available: http://pubdocs.worldbank.org/en/ 165351470911396346/Live-Long-and-Prosper.pdf

2 Pew Reseach Center. Chapter 2: aging in the U.S. and other countries, 2010 to 2050, 2014. Available: https://www.pewresearch. org/global/2014/01/30/chapter-2-aging-in-the-u-s-and-othercountries-2010-to-2050/

3 Prince M, Wimo A, Guerchet M, et al. World Alzheimer report 2015: the global impact of dementia United Kingdom, 2015.

4 Gitlin LN, Marx K, Stanley IH, et al. Translating evidence-based dementia caregiving interventions into practice: State-of-theScience and next steps. Gerontologist 2015;55:210-26.

5 Walter E, Pinquart M. How effective are dementia caregiver interventions? an updated comprehensive meta-analysis. Gerontologist 2019;20:1-11.

6 Arksey H, O'Malley L. Scoping studies: towards a methodological framework. Int J Soc Res Methodol 2005;8:19-32.

7 The World Bank. World bank country and lending groups, 2018. Available: https://datahelpdesk.worldbank.org/knowledgebase/ articles/906519-world-bank-country-and-lending-groups

8 Ali S, Bokharey IZ. Efficacy of cognitive behavior therapy among caregivers of dementia: an outcome study. Pakistan Journal of Psychological Research 2015;30:249-69.

$9 \mathrm{Au} \mathrm{A}$, Li S, Lee K, et al. The coping with caregiving group program for Chinese caregivers of patients with Alzheimer's disease in Hong Kong. Patient Educ Couns 2010;78:256-60.

10 Au A, Wong MK, Leung LM, et al. Telephone-assisted pleasantevent scheduling to enhance well-being of caregivers of people with dementia: a randomised controlled trial. Hong Kong Med $J$ 2014;20:30-3.

11 Au A. Developing volunteer-assisted behavioral activation Teleprograms to meet the needs of Chinese dementia caregivers. Clin Gerontol 2015;38:190-202.

12 Au A, Gallagher-Thompson D, Wong MK, et al. Behavioral activation for dementia caregivers: scheduling pleasant events and enhancing communications. Clin Interv Aging 2015;10:611-9.

13 Chen HM, Huang MF, Yeh YC, et al. Effectiveness of coping strategies intervention on caregiver burden among caregivers of elderly patients with dementia. Psychogeriatrics 2015;15:20-5.

14 Cheng ST, Lau RW, Mak EP, et al. Benefit-finding intervention for Alzheimer caregivers: conceptual framework, implementation issues, and preliminary efficacy. Gerontologist 2014;54:1049-58.

15 Cheng ST, Fung HH, Chan WC, et al. Short-term effects of a gainfocused reappraisal intervention for dementia caregivers: a doubleblind cluster-randomized controlled trial. Am J Geriatr Psychiatry 2016;24:740-50.

16 Cheung KS, Lau BH, Wong PW, et al. Multicomponent intervention on enhancing dementia caregiver well-being and reducing behavioral problems among Hong Kong Chinese: a translational study based on reach II. Int J Geriatr Psychiatry 2015;30:460-9.

17 Chien WT, Lee YM. A disease management program for families of persons in Hong Kong with dementia. Psychiatr Serv 2008;59:433-6.

18 Chien WT, Lee IY. Randomized controlled trial of a dementia care programme for families of home-resided older people with dementia. $J$ Adv Nurs 2011;67:774-87.

19 Chu H, Yang CY, Liao YH, et al. The effects of a support group on dementia caregivers' burden and depression. J Aging Health 2011;23:228-41.

20 Chua J, Pachana NA. Use of a psychoeducational skill training DVD program to reduce stress in Chinese Australian and Singaporean dementia caregivers: a pilot study. Clin Gerontol 2016;39:3-14.

21 Dias A, Dewey ME, D'Souza J, et al. The effectiveness of a home care program for supporting caregivers of persons with dementia in developing countries: a randomised controlled trial from Goa, India. PLoS One 2008;3:e2333. 
22 Fung WY, Chien WT. The effectiveness of a mutual support group for family caregivers of a relative with dementia. Arch Psychiatr Nurs 2002;16:134-44.

23 Hirano A, Suzuki Y, Kuzuya M, et al. Influence of regular exercise on subjective sense of burden and physical symptoms in communitydwelling caregivers of dementia patients: a randomized controlled trial. Arch Gerontol Geriatr 2011;53:e158-63.

24 Hosaka T, Sugiyama Y. A structured intervention for family caregivers of dementia patients: a pilot study. Tokai J Exp Clin Med 1999;24:35-9.

25 Hosaka T, Sugiyama Y. Structured intervention in family caregivers of the demented elderly and changes in their immune function. Psychiatry Clin Neurosci 2003;57:147-51.

26 Huang HL, Shyu YI, Chen MC, et al. A pilot study on a home-based caregiver training program for improving caregiver self-efficacy and decreasing the behavioral problems of elders with dementia in Taiwan. Int J Geriatr Psychiatry 2003;18:337-45.

$27 \mathrm{Kim} \mathrm{H}$. What is the effect of a support program for female family caregivers of dementia on depression? International Journal of BioScience and Bio-Technology 2013;5:39-46.

28 Huang HL, Kuo LM, Chen YS, et al. A home-based training program improves caregivers' skills and dementia patients' aggressive behaviors: a randomized controlled trial. Am J Geriatr Psychiatry 2013;21:1060-70.

29 Kuo L-M, Huang H-L, Liang J, et al. A home-based training program improves Taiwanese family caregivers' quality of life and decreases their risk for depression: a randomized controlled trial. Int J Geriatr Psychiatry 2013;28:504-13.

30 Kuo LM, Huang HL, Hsu WC, et al. Home-based caregiver training: benefits differ by care receivers' dementia diagnosis. Geriatr Nurs 2016;37:376-84.

31 Kuo LM, Huang HL, Liang J, et al. Trajectories of health-related quality of life among family caregivers of individuals with dementia: a home-based caregiver-training program matters. Geriatr Nurs 2017;38:124-32

32 Kuo LM, Huang HL, Liang J, et al. A randomized controlled trial of a home-based training programme to decrease depression in family caregivers of persons with dementia. J Adv Nurs 2017;73:585-98.

33 Kwok T, Wong B, Ip I, et al. Telephone-delivered psychoeducational intervention for Hong Kong Chinese dementia caregivers: a singleblinded randomized controlled trial. Clin Interv Aging 2013;8:1191-7.

34 Kwok T, Au A, Wong B, et al. Effectiveness of online cognitive behavioral therapy on family caregivers of people with dementia. Clin Interv Aging 2014;9:631-6.

35 Lai CK, Wong LF, Liu KH, et al. Online and onsite training for family caregivers of people with dementia: results from a pilot study. Int $J$ Geriatr Psychiatry 2013;28:107-8.

36 Lam LC, Lee JS, Chung JC, et al. A randomized controlled trial to examine the effectiveness of case management model for community dwelling older persons with mild dementia in Hong Kong. Int J Geriatr Psychiatry 2010;25:395-402.
37 Lee YR, Sung KT, Kim YE. Effects of home-based stress management training on primary caregivers of elderly people with dementia in South Korea. Dementia 2012;11:171-9.

38 Senanarong V, Jamjumras $\mathrm{P}$, Harmphadungkit $\mathrm{K}$, et al. A counseling intervention for caregivers: effect on neuropsychiatric symptoms. Int $J$ Geriatr Psychiatry 2004;19:781-8.

39 Wang LQ, Chien WT. Randomised controlled trial of a family-led mutual support programme for people with dementia. J Clin Nurs 2011;20:2362-6.

40 Wang LQ, Chien WT, Lee IY. An experimental study on the effectiveness of a mutual support group for family caregivers of a relative with dementia in mainland China. Contemp Nurse 2012;40:210-24.

41 Yeung CM, Chiu L. Effectiveness of a psychogeriatric carer support group for alleviating carer's distress. Hong Kong Journal Psychiatry 2004;14:24-32.

42 Young KW. A randomized control study on psycho-education group on improving health-related quality of life of Chinese persons with major neurocognitive disorder. Clin Gerontol 2016;39:449-67.

43 Elliott AF, Burgio LD, DeCoster J. Enhancing caregiver health: findings from the resources for enhancing Alzheimer's caregiver health II intervention. J Am Geriatr Soc 2010;58:30-7.

44 Gallagher-Thompson D, Solano N, McGee JS, et al. Coping with caregiving: reducing stress and improving quality of life.: Stanford Univ. School of Medicine and Va Palo Alto Health Care System, 2002. Available: https://www.rosalynncarter.org/research/caregiverintervention-database/coping-with-caregiving-gallagher-thompson/

45 Wu B, Petrovsky DV, Wang J, et al. Dementia caregiver interventions in Chinese people: a systematic review. J Adv Nurs 2019;75:528-42.

46 Prince M, Bryce R, Albanese E, et al. The global prevalence of dementia: a systematic review and metaanalysis. Alzheimers Dement 2013;9:63-75.

47 Bernal G, Sáez-Santiago E. Culturally centered psychosocial interventions. J Community Psychol 2006;34:121-32.

48 Chowdhary N, Jotheeswaran AT, Nadkarni A, et al. The methods and outcomes of cultural adaptations of psychological treatments for depressive disorders: a systematic review. Psychol Med 2014;44:1131-46.

49 Mehta KM, Gallagher-Thompson D, Varghese M, et al. iSupport, an online training and support program for caregivers of people with dementia: study protocol for a randomized controlled trial in India. Trials 2018;19:271.

50 Nguyen TA, Nguyen $\mathrm{H}$, Pham T, et al. A cluster randomized controlled trial to test the feasibility and preliminary effectiveness of a family dementia caregiver intervention in Vietnam. Medicine 2018;97:e12553.

51 Patsopoulos NA. A pragmatic view on pragmatic trials. Dialogues Clin Neurosci 2011;13:217-24.

52 Onken LS, Carroll KM, Shoham V, et al. Reenvisioning clinical science: unifying the discipline to improve the public health. Clin Psychol Sci 2014;2:22-34. 